\title{
Impacto clínico de un grupo de discusión multidisciplinaria en el diagnóstico de fibrosis pulmonar idiopática en Colombia
}

\author{
Clinical impact of a multidisciplinary discussion \\ group on the diagnosis of idiopathic pulmonary \\ fibrosis in Colombia
}

\author{
Mauricio González-García, Emily Rincón-Álvarez, Carolina Pérez-Alvarado, \\ Jacqueline Mugnier-Quijano, Leslie Vargas-Ramirez, Camilo Rodriguez-Cortés, \\ María Fernanda Linares-Contreras, Mauricio Durán Silva • Bogotá, D.C. (Colombia)
}

DOI: https://doi.org/10.36104/amc.2022.2017

\section{Resumen}

Introducción: la fibrosis pulmonar idiopática (FPI) es una enfermedad pulmonar intersticial (EPID) de mal pronóstico, considerada huérfana en Colombia. Un diagnóstico correcto tiene implicaciones para el paciente y los costos de atención. Los grupos de discusión multidisciplinaria (GDM) se consideran el estándar de oro en el diagnóstico. No hay estudios previos en Colombia de la experiencia de un GDM.

Objetivos: evaluar el impacto de un GDM en una institución de cuarto nivel en Bogotá en cambio de diagnóstico de pacientes con EPID y la concordancia entre el diagnóstico inicial y final de FPI.

Material y métodos: pacientes con EPID evaluados entre 2015-2018 por el GDM conformado por neumólogos, radiólogo, patólogo y reumatólogos. Criterios ATS/ERS/JRS/ALAT de diagnóstico de FPI. Descripción del cambio en el diagnóstico y concordancia entre el diagnóstico inicial y del GDM en FPI.

Resultados: de 165 pacientes con EPID se cambió el diagnóstico en 35.2\%. En 77.3\% pacientes con diagnóstico inicial de FPI y en $6.7 \%$ con diagnóstico inicial diferente a FPI el GDM confirmó FPI. Al descartar FPI, los principales diagnósticos fueron neumonitis de hipersensibilidad en fase crónica $(29.4 \%)$ y neumonía intersticial no específica (23.5\%). El índice kappa entre el diagnóstico inicial y final de FPI fue $0.71(0.60-0.82)$

Conclusiones: el GDM en EPID tuvo un importante impacto clínico demostrado por un alto porcentaje de cambió del diagnóstico de remisión. Se descartó el diagnóstico inicial de FPI en un porcentaje significativo de pacientes y se ratificó en un grupo menor sin esta sospecha clínica inicial. (Acta Med Colomb 2022; 47. DOI: https://doi.org/10.36104/amc.2022.2017).

Palabras clave: fibrosis pulmonar idiopática, enfermedad pulmonar intersticial, discusión multidisciplinaria, enfermedad huérfana, diagnóstico, neumonitis de hipersensibilidad.

\footnotetext{
Abstract

Introduction: idiopathic pulmonary fibrosis (IPF) is an interstitial lung disease (ILD) with a poor prognosis, considered an orphan disease in Colombia. An accurate diagnosis has implications for the patient and healthcare costs. Multidisciplinary discussion groups (MDGs) are considered the gold standard for diagnosis. There are no prior studies in Colombia on the experience of an MDG.

Objectives: to evaluate the impact of an MDG in a quaternary care institution in Bogotá on the change in the diagnosis of patients with ILD and the concordance between the initial and final diagnosis of IPF.

Materials and methods: patents with ILD evaluated from 2015-2018 by the MDG made up of pulmonologists, a radiologist, a pathologist and rheumatologists. The ATS/ERS/JRS/ALAT diagnostic criteria for IPF. A description of changes in the diagnosis and the agreement between the initial diagnosis and the MDG diagnosis of IPF.
}

Results: out of 165 patients with ILD, the diagnosis was changed in $32.5 \%$. The MDG confirmed
Dr. Mauricio González-García: Internista, Neumólogo, Epidemiólogo Clínico. Departamento de Investigación; Dra. Emily Rincón-Álvarez: Internista, Neumóloga; Dr. Camilo RodriguezCortés: Internista, Neumólogo; Dra. María Fernanda Linares-Contreras: Internista, Reumatóloga; Dr. Mauricio Durán-Silva: Neumólogo. Fundación Neumológica Colombiana. Dra. Carolina Pérez-Alvarado: Radióloga Departamento de Radiología e Imágenes Diagnósticas; Dra. Jacqueline Mugnier-Quijano: Patóloga Departamento de Patología. Fundación Cardioinfantil. Bogotá, D.C. (Colombia); Dra. Leslie Vargas-Ramírez: Internista, Neumóloga. Fundación Neumológica Colombiana, Bogotá, D.C., Instituto Neumológico del Oriente, Bucaramanga. Colombia.

Correspondencia: Dr. Mauricio GonzálezGarcía. Bogotá, D.C. (Colombia).

E-Mail: mgonzalez@neumologica.org Recibido: 18/IX/2020 Aceptado: 2/VII/2021 
IPF in $77.3 \%$ of patients with an initial diagnosis of ILD and $6.7 \%$ of those with a different initial diagnosis. When IPF was ruled out, the main diagnoses were chronic hypersensitivity pneumonitis $(24.8 \%)$ and nonspecific interstitial pneumonia (23.5\%). The Kappa index between the initial and final IPF diagnoses was $0.71(0.60-0.82)$.

Conclusions: the MDG on ILD had a significant clinical impact evidenced by a high percentage of change in the referral diagnosis. The initial diagnosis of IPF was ruled out in a significant percentage of patients and confirmed in a smaller group which did not have this initial clinical suspicion.

(Acta Med Colomb 2022; 47. DOI: https://doi.org/10.36104/amc.2022.2017).

Key words: idiopathic pulmonary fibrosis, interstitial lung disease, multidisciplinary discussion, orphan disease, diagnosis, hypersensitivity pneumonitis.

\section{Introducción}

La enfermedad pulmonar intersticial (EPID) incluye más de 150 entidades que pueden tener signos, síntomas y presentación radiológica similares, aunque con un enfoque clínico y pronóstico diferentes, lo que hace que sea necesario llegar a un diagnóstico y tratamiento correctos. La EPID se puede separar en aquellas entidades con un trastorno subyacente, como las enfermedades del colágeno, o con una exposición conocida (neumonitis por hipersensibilidad, asbestosis, silicosis) y en las neumonías intersticiales idiopáticas (NII) $(1,2)$.

Las NII son un grupo heterogéneo de enfermedades de curso y pronóstico diverso, siendo la fibrosis pulmonar idiopática (FPI), la más frecuente y la de peor pronóstico. La FPI es una forma específica de neumonía intersticial fibrosante crónica, progresiva, de causa desconocida, que ocurre principalmente en adultos mayores, se limita al pulmón y se asocia con el patrón histopatológico o radiológico de neumonía intersticial usual (NIU) (3). La historia natural es de declinación progresiva de la función pulmonar hasta la muerte por falla respiratoria o secundaria a las comorbilidades, con una media de sobrevida de 3 a 5 años desde el diagnóstico $(1,2,4,5)$.

Desde el 2002, las sociedades científicas internacionales recomiendan un enfoque integrado y dinámico para el diagnóstico de las NII en grupos de discusión multidisciplinaria (GDM) con la participación de neumólogos, radiólogos y patólogos $(1-3,6)$. El diagnóstico del GDM está asociado con mayores niveles de confianza diagnóstica y a un mejor acuerdo interobservador en comparación con los componentes individuales del grupo de forma aislada, por lo que se consideran como el "estándar de oro" para el diagnóstico de la EPID (3, 6-9). Aunque el rendimiento de los GDM puede ser evaluado en términos de precisión en el diagnóstico, la experiencia de los participantes y por estudios de costo efectividad, se acepta que el acuerdo diagnóstico puede utilizarse como sustituto de la precisión diagnóstica (10).

La FPI se acepta como una enfermedad huérfana en Colombia (11) y su correcto diagnóstico tiene implicaciones en el paciente y en los costos de atención. Debido a que no hay estudios en Colombia, nuestro objetivo fue evaluar el impacto de un GDM en una institución de $4^{\circ}$ nivel en Bogotá en el cambio de diagnóstico de pacientes con EPID y FPI.

\section{Material y métodos}

\section{Pacientes y criterios de diagnóstico}

Se incluyeron en forma consecutiva todos los pacientes con EPID evaluados entre enero de 2015 y diciembre de 2018 por el GDM. Se utilizaron los criterios de la Sociedad Americana del tórax (ATS) y la Sociedad de Enfermedad Respiratoria Europea (ERS) para el diagnóstico y clasificación de las EPID $(1,2)$ y los criterios conjuntos de la ATS, ERS, JRS (Sociedad de Enfermedad Respiratoria Japonesa) y ALAT (Sociedad Latinoamericana del Tórax) para diagnóstico de FPI (3). Este estudio fue aprobado por el Comité de Ética e Investigación de la institución y todos los pacientes firmaron un consentimiento para la utilización de la información clínica.

\section{Grupo de discusión multidisciplinario}

El GDM fue creado en 2104 según recomendaciones de las guías internacionales $(3,6,12)$. Está conformado por médicos especialistas en neumología, radiología, patología y reumatología con experiencia en EPID y se realiza de dos a cuatro reuniones al mes. Se hace revisión de la historia clínica con énfasis en la búsqueda de factores de riesgo para enfermedad pulmonar, exposiciones ambientales y posibles enfermedades sistémicas. Como requisitos mínimos se requiere la presentación de las pruebas inmunológicas relacionadas con EPID, las pruebas de función pulmonar (espirometría y difusión de monóxido de carbono) y la tomografía del tórax que deben haber sido realizadas en los últimos tres meses. También se presentan exámenes complementarios como gases arteriales, caminata de seis minutos, tomografías previas para comparación o resultados de broncoscopia. En caso de biopsias pulmonares, se revisan los hallazgos de la patología. En caso de que se soliciten nuevos exámenes por el GDM, se realiza una nueva presentación para establecer un diagnóstico final. Se registra el diagnóstico del médico tratante previo al GDM y el diagnóstico definitivo de consenso.

\section{Análisis estadístico}

Se realizó la descripción del porcentaje de cambio en el diagnóstico de la EPID en general y de la FPI. La descripción de las características clínicas y funcionales de los pacientes de FPI se realizó con promedios y desviaciones estándar para 
las variables cuantitativas y proporciones para las variables cualitativas. Para la FPI se evaluó concordancia entre el diagnóstico previo al GDM y el diagnóstico definitivo del GDM usando el coeficiente de Kappa, como es usual en este tipo de estudios. Se utilizó el software estadístico SPSS 15.0.

\section{Resultados}

Se incluyeron 165 pacientes con EPID, el 55.2\% hombres y con edad promedio de $69.0 \pm 12.4$ años. La frecuencia de los diagnósticos de remisión al GDM se muestran en la Tabla 1.

Hubo cambio de diagnóstico por el GDM en 58 del total de 165 pacientes evaluados con EPID (35.2\%) y en 17 de los $75(22.7 \%)$ que tenían diagnóstico inicial de FPI. En seis pacientes de los 90 (6.7\%) sin diagnóstico inicial de FPI se confirmó este diagnóstico por el GDM. Los diagnósticos finales más frecuentes fueron FPI, EPID asociada a enfermedad del tejido conectivo (ETC), neumonitis de hipersensibilidad en fase crónica $(\mathrm{NH})$ y neumonía intersticial no específica (NINE) (Figura 1).

La concordancia entre el diagnóstico inicial y final por el GDM de FPI evaluado por el índice kappa fue 0.71 (0.600.82) (Tabla 2). De los 165 pacientes, 64 (38.8\%) tuvieron diagnóstico final de FPI por el GDM. En los 17 pacientes en quienes se descartó la FPI, el diagnóstico final más frecuente

Tabla 1. Diagnósticos de EPID de remisión al GDM $(N=165)$.

\begin{tabular}{|l|c|}
\hline \multicolumn{1}{|c|}{ Edad, años } & $\mathbf{6 9 . 0 \pm 1 2 . 4}$ \\
\hline Hombres, \% & $91(55.2)$ \\
\hline - Diagnósticos de remisión al GDM & \\
- Fibrosis pulmonar idiopática & $75(45.5)$ \\
- Neumonía intersticial no específica & $29(17.6)$ \\
- EPID asociada a enfermedad del colágeno & $22(13.3)$ \\
- Neumonitis de hipersensibilidad & $12(7.3)$ \\
- Sarcoidosis & $6(3.6)$ \\
- Neumonía organizada & $3(1.8)$ \\
- Neumonía intersticial linfoide & $3(1.8)$ \\
- Neumoconiosis & $3(1.8)$ \\
- Neumonitis por medicamentos & $3(1.8)$ \\
- Osificación pulmonar & $3(1.8)$ \\
- Otros & $8(4.8)$ \\
\hline GMD: grupo de discusión multidisciplinaria. & \\
Valores como promedio \pm DE o N (\%). & \\
\hline
\end{tabular}

Tabla 2. Concordancia entre diagnóstico inicial y el diagnóstico final del GDM.

\begin{tabular}{|l|l|c|c|c|}
\hline \multicolumn{2}{|c|}{} & \multicolumn{2}{|c|}{ Diagnóstico GMD } & \multirow{2}{*}{ Total } \\
\cline { 3 - 5 } & FPI & No FPI & \\
\hline \multirow{2}{*}{ Diagnóstico inicial } & FPI & 58 & 17 & 75 \\
\cline { 2 - 5 } & No FPI & 6 & 84 & 90 \\
\hline \multirow{2}{*}{ Total } & 64 & 102 & 165 \\
\hline $\begin{array}{l}\text { GMD: grupo de discusión multidisciplinaria; FPI: fibrosis pulmonar idiopática. } \\
\text { Kappa=0.71 (0.60-0.82). }\end{array}$ \\
\hline
\end{tabular}

fue neumonitis de hipersensibilidad en fase crónica en cinco (29.4\%), NINE en cuatro (23.5\%) y neumonía intersticial no clasificable (NINC) en tres (17.6\%).

Los pacientes con diagnóstico final de FPI por el GDM fueron principalmente hombres $(75.0 \%)$ y con antecedente de tabaquismo (75\%). Se realizó biopsia pulmonar en 13 de estos 64 pacientes (20.3\%). Funcionalmente tuvieron disminución de la capacidad vital forzada, de la difusión de monóxido de carbono, hipoxemia y diferencia alveoloarterial de oxígeno alta (Tabla 3).

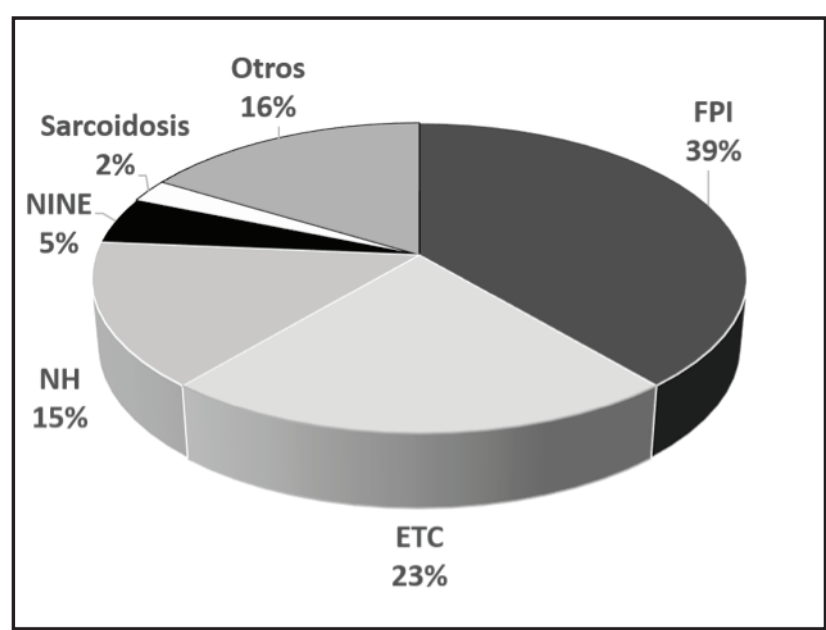

Figura 1. Diagnósticos finales por el GDM. GMD: grupo de discusión multidisciplinaria NINE: neumonía intersticial no específica: NH: neumonitis de hipersensibilidad; ETC: enfermedad pulmonar intersticial asociada a enfermedad del tejido conectivo.

Tabla 3. Características de los pacientes con FPI $(N=64)$.

\begin{tabular}{|c|c|}
\hline Edad, años & $68.4 \pm 10.9$ \\
\hline Hombres & $48(75.0)$ \\
\hline $\mathrm{IMC}, \mathrm{Kg} / \mathrm{m}^{2}$ & $26.8 \pm 4.1$ \\
\hline Tabaquismo & $47(73.4)$ \\
\hline Biopsia pulmonar & $13(20.3)$ \\
\hline CVF, \% predicho & $76.0 \pm 17.2$ \\
\hline $\mathrm{VEF}_{1}, \%$ predicho & $78.8 \pm 18.3$ \\
\hline $\mathrm{VEF}_{1} / \mathrm{CVF}$ & $82.0 \pm 8.7$ \\
\hline $\mathrm{DL}_{\mathrm{CO}}, \%$ predicho & $50.8 \pm 13.9$ \\
\hline $\mathrm{PaCO}_{2}, \mathrm{mmHg}$ & $34.5 \pm 3.8$ \\
\hline $\mathrm{PaO}_{2}, \mathrm{mmHg}$ & $53.4 \pm 9.3$ \\
\hline $\mathrm{SaO}_{2}, \%$ & $87.2 \pm 5.3$ \\
\hline $\mathrm{P}(\mathrm{A}-\mathrm{a}) \mathrm{O}_{2}, \mathrm{mmHg}$ & $16.0 \pm 8.3$ \\
\hline \multicolumn{2}{|c|}{$\begin{array}{l}\text { FPI: fibrosis pulmonar idiopática.IMC: índice de masa corporal; } \mathrm{CVF} \text { : capacidad vital } \\
\text { forzada; } V E F_{1}: \text { volumen espiratorio forzado en el primer segundo; } \mathrm{DL} \mathrm{Co}_{\mathrm{Co}} \text { : difusión de } \\
\text { monóxido de carbono; } \mathrm{PaCO}_{2}: \text { presión arterial de bióxido de carbono; } \mathrm{PaO}_{2}: \text { presión } \\
\text { arterial de oxígeno; } \mathrm{SaO}_{2}: \text { saturación arterial de oxígeno: } \mathrm{P}(\mathrm{A}-\mathrm{a}) \mathrm{O}_{2}: \text { diferencia alveolo- } \\
\text { arterial de oxígeno. } \\
\text { Valores como promedio } \pm \mathrm{DE} \text { o } \mathrm{N}(\%) \text {. }\end{array}$} \\
\hline
\end{tabular}




\section{Discusión}

Este GDM centrado en la discusión de pacientes con EPID tuvo un importante impacto clínico demostrado por el cambio del diagnóstico en un alto porcentaje de los de pacientes evaluados. Específicamente en la FPI, se descartó este diagnóstico en un porcentaje significativo de pacientes y se ratificó en un grupo menor sin esta sospecha clínica inicial, lo que conlleva a un cambio de la conducta médica para la atención apropiada de estos pacientes.

Estudios previos han demostrado el cambio de diagnóstico por el GDM en un porcentaje alto de casos. En un estudio realizado en dos centros especializados en EPID que incluyó 90 pacientes, se reportó cambio en el diagnóstico de EPID en 53\% de todos los casos y en 37\% de los pacientes referidos con diagnóstico de FPI (13). En otro estudio, el GDM alcanzó un diagnóstico preciso en $88 \%$ de los casos y el diagnóstico fue cambiado en 58 pacientes (64\%) (14). En un estudio retrospectivo con 938 casos el GDM llegó a un diagnóstico definitivo en $80.5 \%$ de los casos y hubo cambio de diagnóstico en $41.9 \%$ (15).

En este estudio mostramos que el cambio en el diagnóstico por el GDM se presentó en $35 \%$ de los casos, aunque alto, inferior a lo reportado en la literatura (13-15) y que la concordancia entre el diagnóstico previo al GDM y el diagnóstico final por le GDM no fue tan baja (0.71). Este porcentaje de cambio de diagnóstico menor a lo reportado y la concordancia mostrada pueden ser explicados porque el diagnóstico previo al GDM fue realizado por un médico especialista neumólogo y no por médicos internistas o generales como en varios de los estudios mencionados.

En el grupo total de EPID, los diagnósticos finales más frecuentes del GDM fueron la FPI, la EPID relacionada a ETC y la NH similar a lo descrito en grandes series $(9,16)$. Tanto la ETC y la NH son diagnósticos diferenciales de la FPI y se recomienda descartar estas entidades durante la evaluación del paciente con sospecha de FPI $(3,6,16,17)$. En 3.6\% de los pacientes de nuestro estudio, el diagnóstico final fue NINC, menor a lo reportado de $5-15 \%$ en otros estudios $(9,18)$. A diferencia de otras series de EPID, en este estudio tuvimos pocos casos de neumoconiosis lo cual es explicado por qué en nuestra institución estos pacientes son presentados en la Junta de Neumología Ocupacional y no en el GDM de EPID.

En un porcentaje alto (22.7\%) se descartó el diagnóstico inicial de FPI, lo que tiene alto impacto para el pronóstico, el enfoque clínico y terapéutico y por lo tanto en el costo de la atención en salud de estos pacientes. La FPI de una enfermedad con una alta carga en términos de salud y costos $(19,20)$ y se conoce que el enfoque multidisciplinario de estos pacientes con un diagnóstico más preciso y temprano puede llevar a mejores desenlaces clínicos $(13,21,22)$.

En el contexto Latinoamericano con diferencias entre nuestros países en términos sociales, económicos y de sistemas de salud, se reconoce la importancia de la aplicación de las guías de manejo de la FPI. Se ha resaltado la importancia del fortalecimiento de GDM y centros de remisión de EPID, conociendo que el rendimiento diagnóstico de estas enfermedades está determinado por la experiencia de estos grupos médicos, lo que finalmente debe llevar a la optimización de los recursos de salud y uso racional de tratamientos de alto costo de estas enfermedades (23).

En los 17 pacientes en quienes se descartó el diagnóstico de FPI los diagnósticos finales más frecuentes fueron NH (29.4\%) y la neumonía intersticial no específica como ha sido reportado previamente. Es importante resaltar que tres (17.6\%) de estos pacientes en quienes se descartó FPI, tuvieron un diagnóstico final de NINC, a pesar de estudios completos de imágenes, historia exhaustiva de exposiciones y estudios complementarios para descartar ETC.

Como se ha descrito en otros estudios, los pacientes con diagnóstico final de FPI por el GDM fueron principalmente hombres, con antecedente de tabaquismo y con un comportamiento funcional característico de disminución de la capacidad vital forzada, la difusión de monóxido de carbono e hipoxemia. La realización de biopsia pulmonar en $20.3 \%$ de los pacientes fue similar a lo descrito en los estudios pivótales con los que se aprobó la pirfenidona y en nintedanib para uso en FPI $(24,25)$ y está de acuerdo a las recomendaciones internacionales en las cuales, en presencia de un cuadro clínico sugestivo, la tomografía del tórax puede ser diagnóstica de la enfermedad $(3,6)$.

La composición de nuestro GDM es similar a lo sugerido y reportado en la literatura $(3,6,26)$. En una encuesta a 10 centros de expertos en EPID de Europa, Norte América y Australia se reportó la asistencia en 100\% de los grupos de neumólogos, radiólogos y patólogos (27). En esta misma encuesta sólo se reportó asistencia de reumatólogos en 30\% de estos centros. En otro estudio con participación de más centros, la asistencia del radiólogo y el neumólogo fue una característica común a todos los grupos, mientras que la asistencia del reumatólogo y el patólogo fue más probable en grupos de centros académicos (28).

Aunque en varias publicaciones se considera que la participación del reumatólogo debería centrarse a la evaluación clínica de los pacientes y no en su participación directa en los GDM, algunos estudios resaltan la importancia de su participación en los GDM. En grupos expertos se ha informado la realización de diagnósticos nuevos de EPID relacionada a ETC en aproximadamente $10 \%$ de los pacientes (9), reclasificación de pacientes inicialmente considerados como FPI o la necesidad de menos exámenes invasivos adicionales una vez establecido el diagnóstico de una posible ETC (29-31). En nuestra experiencia se realizaron 15 diagnósticos nuevos de EPID relacionada a ETC y el diagnóstico de EPID relacionada a ETC fue el segundo diagnóstico definitivo más frecuente $(22.4 \%)$ después de la FPI, resaltando la importancia de un reumatólogo en el proceso diagnóstico de estas enfermedades.

Al comparar la organización y estructura de nuestro GDM tenemos características similares a otros grupos mul- 
tidisciplinarios. Estas son un grupo exclusivo para casos de EPID, tener una frecuencia de reuniones una a dos veces a la semana, de duración de 60 minutos y tener como requisito para la presentación de los casos exámenes mínimos como la tomografía del tórax, las pruebas de función pulmonar, la serología para estudio de las ETC o las biopsias pulmonares o en lavado broncoalveolar en caso de requerirlos (27).

Aunque el GDM se considera el "estándar de oro" para el diagnóstico de la EPID, existen algunas limitaciones para el desempeño de estos grupos. En un estudio que evaluó la concordancia interobservador para los criterios de NIU en la tomografía utilizando las guías de las sociedades científicas (3), se reportó solamente un acuerdo moderado entre radiólogos, independientemente de su grado de experiencia (32). También se ha demostrado que los médicos con mayor experiencia de centros académicos tienen mayor acuerdo en el diagnóstico de la FPI que aquellos de centros no académicos (33). Adicionalmente, se ha reportado que el nivel de acuerdo para el diagnóstico de FPI es mayor que para otras enfermedades frecuentes como la NH y la NINE, entidades de diagnóstico diferencial frecuente con la FPI (9).

Como fortalezas de este estudio resaltamos que se trata del primer trabajo que muestra la experiencia de un GDM en EPID en Colombia con un porcentaje alto de cambio de diagnóstico, lo que tiene alto impacto clínico para el manejo de estos pacientes. Nuestro GDM tiene una estructura claramente definida con profesionales de diferentes especialidades médicas con experticia en el enfoque del paciente EPID y una exigencia de requisitos mínimos de presentación de los casos que permite el estudio completo de los pacientes. Siendo al EPID y particularmente la FPI enfermedades de baja prevalencia, consideramos que la muestra del estudio es significativa y que soporta las conclusiones obtenidas en el estudio.

Como debilidad principal, similar a lo reportado en la literatura para los GDM, está la falta de verificación de los resultados del GDM, para lo cual se requiere seguimiento de los pacientes a mediano y largo plazo con desenlaces clínicos para confirmar con más certeza los diagnósticos finales por el GDM. Adicionalmente es importante la realización de estudios de costos para establecer el resultado económico derivado de los cambios de diagnóstico y conductas de estos pacientes, lo cual no fue evaluado en el presente trabajo.

En conclusión, nuestro GDM tuvo un importante impacto clínico demostrado por el cambio del diagnóstico en un alto porcentaje de los de pacientes con EPID evaluados. Específicamente en la FPI, se descartó este diagnóstico en un porcentaje significativo de pacientes y se ratificó en un grupo menor sin esta sospecha clínica inicial, lo que conlleva a un cambio de la conducta médica para la atención apropiada de estos pacientes.

\section{Referencias}

1. American Thoracic Society/European Respiratory Society International Multidisciplinary Consensus Classification of the Idiopathic Interstitial Pneumonias. This joint statement of the American Thoracic Society (ATS), and the European Respiratory Society (ERS) was adopted by the ATS board of directors, June 2001 and by the ERS Executive Committee, June 2001. Am J Respir Crit Care Med. 2002;165(2):277-304.

2. Travis WD, Costabel U, Hansell DM, King TE, Jr., Lynch DA, Nicholson AG, et al. An official American Thoracic Society/European Respiratory Society statement: Update of the international multidisciplinary classification of the idiopathic interstitial pneumonias. Am J Respir Crit Care Med. 2013;188(6):733-48.

3. Raghu G, Collard HR, Egan JJ, Martinez FJ, Behr J, Brown KK, et al. An official ATS/ERS/JRS/ALAT statement: idiopathic pulmonary fibrosis: evidencebased guidelines for diagnosis and management. Am J Respir Crit Care Med. 2011;183(6):788-824.

4. American Thoracic Society. Idiopathic pulmonary fibrosis: diagnosis and treatment. International consensus statement. American Thoracic Society (ATS), and the European Respiratory Society (ERS). Am J Respir Crit Care Med. 2000;161(2 Pt 1):646-64

5. Gonzalez-Garcia M, Chamorro J, Jaramillo C, Casas A, Maldonado D. Survival of patients with idiopathic pulmonary fibrosis at the altitude of Bogota (2640 m). Acta Med Colomb. 2014;39(1):15-20.

6. Raghu G, Remy-Jardin M, Myers JL, Richeldi L, Ryerson CJ, Lederer DJ, et al. Diagnosis of Idiopathic Pulmonary Fibrosis. An Official ATS/ERS/JRS/ALAT Clinical Practice Guideline. Am J Respir Crit Care Med. 2018;198(5):e44-e68.

7. Flaherty KR, King TE, Jr., Raghu G, Lynch JP, 3rd, Colby TV, Travis WD, et al. Idiopathic interstitial pneumonia: what is the effect of a multidisciplinary approach to diagnosis? Am J Respir Crit Care Med. 2004;170(8):904-10.

8. Thomeer M, Demedts M, Behr J, Buhl R, Costabel U, Flower CD, et al. Multidisciplinary interobserver agreement in the diagnosis of idiopathic pulmonary fibrosis. The European Respiratory Journal. 2008;31(3):585-91.

9. Walsh SLF, Wells AU, Desai SR, Poletti V, Piciucchi S, Dubini A, et al. Multicentre evaluation of multidisciplinary team meeting agreement on diagnosis in diffuse parenchymal lung disease: a case-cohort study. Lancet Respir Med. 2016;4(7):557-65.

10. Walsh SLF. Multidisciplinary evaluation of interstitial lung diseases: current insights: Number 1 in the Series "Radiology" Edited by Nicola Sverzellati and Sujal Desai. European respiratory review: an official journal of the European Respiratory Society. 2017;26(144).

11. Ministerio de Salud y Protección Social. Resolución 2048 de 2015 Bogota2015: https://www.minsalud.gov.co/Normatividad_Nuevo/Resoluci\%C3\%B3n\%20 2048\%20de\%202015.pdf.

12. NICE. Idiopathic pulmonary fibrosis in adults: diagnosis and management National Institute for Health and Care Excellence: Clinical Guidelines. London: National Institute of Health and Care Excellence; 2017.

13. Jo HE, Glaspole IN, Levin KC, McCormack SR, Mahar AM, Cooper WA, et al. Clinical impact of the interstitial lung disease multidisciplinary service. Respirology. 2016;21(8):1438-44.

14. Guler SA, Berezowska SA, Christe A, Geiser T, Funke-Chambour M. Multidisciplinary discussion for diagnosis of interstitial lung disease in real life. Swiss medical weekly. 2016;146:w14318.

15. De Sadeleer LJ, Meert C, Yserbyt J, Slabbynck H, Verschakelen JA, Verbeken EK, et al. Diagnostic Ability of a Dynamic Multidisciplinary Discussion in Interstitial Lung Diseases: A Retrospective Observational Study of 938 Cases. Chest. 2018;153(6):1416-23.

16. Lederer DJ, Martinez FJ. Idiopathic Pulmonary Fibrosis. $N$ Engl J Med 2018;378(19): 1811-23.

17. Richeldi L, Collard HR, Jones MG. Idiopathic pulmonary fibrosis. Lancet. 2017;389(10082):1941-52.

18. Skolnik K, Ryerson CJ. Unclassifiable interstitial lung disease: A review. Res pirology. 2016;21(1):51-6.

19. Collard HR, Chen SY, Yeh WS, Li Q, Lee YC, Wang A, et al. Health care utilization and costs of idiopathic pulmonary fibrosis in U.S. Medicare beneficiaries aged 65 years and older. Annals of the American Thoracic Society. 2015;12(7):981-7.

20. Raimundo K, Chang E, Broder MS, Alexander K, Zazzali J, Swigris JJ. Clinical and economic burden of idiopathic pulmonary fibrosis: a retrospective cohort study. BMC Pulm Med. 2016;16:2.

21. Aiello M, Bertorelli G, Bocchino M, Chetta A, Fiore-Donati A, Fois A, et al The earlier, the better: Impact of early diagnosis on clinical outcome in idiopathic pulmonary fibrosis. Pulm Pharmacol Ther. 2017;44:7-15.

22. Molina-Molina M, Aburto M, Acosta O, Ancochea J, Rodriguez-Portal JA, Sauleda J, et al. Importance of early diagnosis and treatment in idiopathic pulmonary fibrosis. Expert Rev Respir Med. 2018;12(7):537-9.

23. Castillo D, Enghelmayer JI. Can Clinical Guidelines on Idiopathic Pulmonary Fibrosis be Applied in Ibero-America? The Need to Establish Centers of Reference. Archivos de bronconeumologia. 2020;56(3):135-6. 
24. Richeldi L, du Bois RM, Raghu G, Azuma A, Brown KK, Costabel U, et al. Efficacy and safety of nintedanib in idiopathic pulmonary fibrosis. $N$ Engl J Med. 2014;370(22):2071-82

25. King TE, Jr., Bradford WZ, Castro-Bernardini S, Fagan EA, Glaspole I, Glassberg MK, et al. A phase 3 trial of pirfenidone in patients with idiopathic pulmonary fibrosis. N Engl J Med. 2014;370(22):2083-92.

26. Prasad JD, Mahar A, Bleasel J, Ellis SJ, Chambers DC, Lake F, et al. The interstitial lung disease multidisciplinary meeting: A position statement from the Thoracic Society of Australia and New Zealand and the Lung Foundation Australia. Respirology. 2017;22(7):1459-72.

27. Jo HE, Corte TJ, Moodley Y, Levin K, Westall G, Hopkins P, et al. Evaluating the interstitial lung disease multidisciplinary meeting: a survey of expert centres. BMC Pulm Med. 2016;16:22.

28. Richeldi L, Launders N, Martinez F, Walsh SLF, Myers J, Wang B, et al. The characterisation of interstitial lung disease multidisciplinary team meetings: a global study. ERJ Open Res. 2019;5(2).
29. Chartrand S, Swigris JJ, Peykova L, Chung J, Fischer A. A Multidisciplinary Evaluation Helps Identify the Antisynthetase Syndrome in Patients Presenting as Idiopathic Interstitial Pneumonia. J Rheumatol. 2016;43(5):887-92.

30. Ferri C, Manfredi A, Sebastiani M, Colaci M, Giuggioli D, Vacchi C, et al Interstitial pneumonia with autoimmune features and undifferentiated connective tissue disease: Our interdisciplinary rheumatology-pneumology experience, and review of the literature. Autoimmun Rev. 2016;15(1):61-70.

31. Levi Y, Israeli-Shani L, Kuchuk M, Epstein Shochet G, Koslow M, Shitrit D. Rheumatological Assessment Is Important for Interstitial Lung Disease Diagnosis. J Rheumatol. 2018;45(11):1509-14.

32. Walsh SL, Calandriello L, Sverzellati N, Wells AU, Hansell DM, Consort UIPO. Interobserver agreement for the ATS/ERS/JRS/ALAT criteria for a UIP pattern on CT. Thorax. 2016;71(1):45-51.

33. Walsh SLF, Maher TM, Kolb M, Poletti V, Nusser R, Richeldi L, et al. Diagnostic accuracy of a clinical diagnosis of idiopathic pulmonary fibrosis: an international case-cohort study. The European Respiratory Journal. 2017;50(2). 